\title{
Eating Disorders in a Multiple Sclerosis Clinical Population and its Association with Social Anxiety
}

\author{
Shahla Mohamadirizi ${ }^{1^{*}}$, Vahid Shaygannejad ${ }^{2}$, Soheila Mohamadirizi ${ }^{3}$ and Marjan Mohamadirizi ${ }^{4}$ \\ ${ }^{1}$ Departments of Nursing, School of Nursing and Midwifery, University of Medical Sciences, Isfahan, Iran \\ ${ }^{2}$ Department of Neurology, School of Medicine, Isfahan Neuroscience Research Center (INRC), Iran \\ ${ }^{3}$ Department of Midwifery, School of Nursing and Midwifery, Isfahan University of Medical Sciences, Isfahan, Iran
}

*Corresponding author: Mohamadirizi S, Departments of Nursing, School of Nursing and Midwifery, University of Medical Sciences, Isfahan, Iran, Tel: + 09103142630; E-mail: mohamadirizi@yahoo.com

Received date: July 10, 2016; Accepted date: September 10, 2016; Published date: September 17, 2016

Copyright: ( ) 2016 Mohamadirizi S, et al. This is an open-access article distributed under the terms of the Creative Commons Attribution License, which permits unrestricted use, distribution and reproduction in any medium, provided the original author and source are credited.

\begin{abstract}
Context: Social anxiety and eating disorders have demonstrated high comorbidity in a Multiple Sclerosis Clinic Population. However, social anxiety has not been directly studied with respect to eating disorders. This study, therefore, was designed to determine the relationship between social anxiety and eating disorders in a multiple sclerosis clinic population.
\end{abstract}

Methods and materials: This was a cross-sectional study which was conducted in Kashani Hospital affiliated with Isfahan University of Medical Sciences, Iran, in 2013. 210 adult patients who suffered from multiple sclerosis were selected and completed the Demographic Characteristics Questionnaire, Eating Disorder Examination Questionnaire (EDE-Q) and Anxiety Disorder Inventory. SPSS Version 16 software was used to conduct statistical tests including t-test, ANOVA and Pearson correlation.

Results: The results showed that mean and standard deviation of the eating disorder and social anxiety scores were $1.2 \pm 0.15$ and $17.9 \pm 8.5$, respectively. Also, $7.2 \%$ of multiple sclerosis patients had eating disorder and $39.1 \%$ social anxiety disorder. There was a significant positive correlation between the social anxiety score and eating disorder score $(r=0.4, p \leq 0.05)$.

Conclusion: Our study indicated a significant level of social anxiety and eating disorders in people with multiple sclerosis. Eating disorders in multiple sclerosis patients was strongly related with social anxiety. The findings from this study can assist health care team to pay more attention to social anxiety and eating disorders in people with multiple sclerosis, and also consider their relationship in their evaluations.

Keywords: Eating disorder; Social anxiety; Multiple sclerosis

\section{Introduction}

Multiple sclerosis (MS) is a chronic, progressive, and central nervous system-demyelinating disease, with a different course and uncertain prognosis. Multiple sclerosis is most common among 20-40 year old people. In previous decade, U.S. National Multiple Sclerosis Society announced that approximately 572,000 people had multiple sclerosis worldwide, and 200 new cases were diagnosed each week. According to statistics by the Iran Multiple Sclerosis Society, approximately 40,000 people have multiple sclerosis in the country. In Isfahan Province, prevalence estimates are approximately 35.5 per 100,000 people and about 1400 in total population of 3,923,255 people, so Isfahan can be recognized as a region of medium to high risk of multiple sclerosis. Considering increasing prevalence of multiple sclerosis, its high mortality rates, high costs and various problems faced by patients with multiple sclerosis, it is necessary to pay attention to multiple sclerosis patients and try to alleviate its problems and symptoms [1].

It is worth to mention that multiple sclerosis patients experience a wide range of neuropsychiatric disorders, in addition to physical problems associated with the disease; however, most patients adapt to these psychological aspects, which sometimes result from chronic medical problems. Eating disorders and social-anxiety disorders are examples of these mental disorders [2].

Debilitating disorders characterized by increasing preoccupation with weight, food and body shape are called eating disorders. Eating disorders influence public health and their prevalence has increased in recent decades. Available statistics represents increased eating disorder in the public. Progression of eating disorder, however, is more considerable in people with chronic disease, due to chronic disease process and subsequent consequences [3]. In fact, people with chronic diseases are more likely to be affected by eating disorder than their healthy counterparts [4]. Studies of eating disorder behaviours in chronic diseases have also shown that these symptoms are progressing over time and gradually aggravating.

According to psychiatric disorders, eating disorders are divided into three general categories: anorexia nervosa, bulimia nervosa, and eating disorder not otherwise specified [5]. These disorders start as mild states (such as negative eating attitude), and then converted to severe and clinical states (such as anorexia nervosa and bulimia nervosa), so it is of high importance to recognize people at risk and factors causing them. When examining these diseases, one of issues considered is 
negative eating attitudes, which includes abnormal views on current and ideal weight; mental image of body and eating behaviours; preoccupation with food contents and their metabolism in the body; and the use of specific methods of the excretion of food from the body [6].

In general, eating disorder is a biopsychosocial disease associated with different factors including cultural, social and psychological factors. According to different studies, psychological disorders like depression and types of anxiety are associated with disorders related to nutrition. Although the nature of this association is not fully clear yet, the causes of nutritional disorders emphasize an important role of types of psychiatric disorders, based on most theories. Most epidemiological and clinical studies have shown that people with a type of eating disorders are affected by one or more anxiety disorders. In fact, anxiety is a predisposition and main factor in developing eating disorders [7]. In this respect, in his study, Walter noted that one half of people with eating disorder symptoms had one or more anxiety disorders, which among their $20 \%$ had social anxiety [8].

Social anxiety is a broad term to describe behavior related to embarrassment and fear of social situations, which ranges from mild to severe [9]. Social anxiety is characterized by considerable and continuous fear of one or more social situations in which patients feel that their actions and behaviors are judged and avoid social situations and interpersonal relationships. Discomfort in social situations, staring, and tendency to silence in front of others are some terms of social anxiety [10]. In fact, social anxiety is a level of fear or distress causing morbid level, which develops as continuous fear of one or more social situations against unfamiliar individuals.

Fear of negative evaluation by others is considered as first attribute of social anxiety. Fear and anxiety are accompanied by body dissatisfaction and abnormal eating attitudes [11]. In fact, people having negative thoughts about their bodies are more concerned about others' opinions on their bodies [12]. The severity of these negative thoughts and attitudes may result in changes to their behavior; subsequently, they would avoid social situations in which their bodies may be exposed to public view. These people try to promote their body size and shape by dieting and not eating food [9].

Considering the probability of comorbidity of social anxiety with eating disorders in patients with multiple sclerosis (MS); due to the fact that few scientific studies have been conducted to examine the relationship between social anxiety disorders and eating disorders in patients with multiple sclerosis regarding disease conditions; that social anxiety disorders and eating disorders have different prevalence in different societies and cultures; because of the existence of contradicting results about the relationship between the two disorders including Celikel's study in which there is a positive correlation between nutritional disorders, including eating attitude, and social anxiety, and Bas's study in which there is no correlation between eating attitude and social anxiety $[13,14]$; and that such a study has not been reported in Iran so far, the researcher tried to examine the relationship between social anxiety disorders and eating disorders in patients with multiple sclerosis (MS) visiting Kashani Hospital in Isfahan City in 2013.

\section{Procedure}

The present research is a cross-sectional study of correlation type which was conducted on 210 patients with multiple sclerosis visiting the multiple sclerosis Clinic of Kashani Hospital affiliated with Isfahan
University of Medical Sciences in 2013. First, presenting the center with the letter of introduction and obtaining their permission, qualified people were selected based on the subject selection checklist, and, then, the objective of the research was explained to them if there were all inclusion criteria and the consent form was completed.

In the present study, inclusion criteria [being Iranian, being Muslim, being resident in Isfahan City, having physical and mental ability to participate in the study, having written consent to participate in the study, etc.] and exclusion criteria [the use of medications affecting mind, having medical disease (thyroid, diabetes, hypertension, cardiovascular disease, renal diseases, neurological diseases,...), having specific diet (Herbal therapy, medication therapy,...), having eating disorder (anorexia, bulimia) in one of family members, being a professional athlete, etc.] checked.

Research tools included Demographic Characteristics Questionnaire (10 items), standard Social Anxiety Inventory (17 items) and Eating Disorder Examination Questionnaire (EDE-Q) (10 items), which were completed by subjects. The Demographic Characteristics Questionnaire included 10 items, and its validity was determined by content validity. These tools were prepared by studying the most recent books and papers in the field and given to experts and practitioners to evaluate.

The social anxiety Inventory was used to diagnose social anxiety disorder. The self-rating Social Anxiety Inventory includes 17 items and is rated on five-point Likert scale from 0 (Not at all) to 4 (Extremely). Its cut-off point is 19, and in total its score is between 0 and 68. A score higher than 19 represents the existence of social anxiety. The self-rating inventory was designed by Connor et al. to evaluate social phobia. It was evaluated by one-way analysis of variance in three groups in terms of psychometric properties in Iran, which showed significant mean differences among the groups [15]. In another research, Momeni used the Social Anxiety Inventory and reported total Cronbach's alpha of 0.88 , first-half Cronbach's alpha of 0.81 , second-half Cronbach's alpha of 0.77 , correlation between the two halves of 0.77 , validity and reliability coefficient of 0.87 by the BrownSpearman test $[16,17]$. In addition, Afzali used the inventory and reported it as a valid and reliable one [18].

The Eating Disorder Examination Questionnaire (EDE-Q) was used to obtain information on eating disorder. The Eating Disorder Examination Questionnaire (EDE-Q) includes 28 items and 4 subscales (weight concern, shape concern, eating concern and dietary restraint) and is rated on seven-point Likert scale from 0 (Not at all) to 6 (Always). A score equal to and higher than 4 on the total test and also on its subscales represents the existence of eating disorder on the dimension or on the total test. The questionnaire is a valid and reliable tool which is confirmed in studies conducted in Western countries such that its reliability has been reported about 0.9 in different studies $[19,20]$.

\section{Ethical Considerations}

In this study, the tests and questionnaire designers were kept anonymous, and informed consent forms were obtained from the participants; the subjects were assured about the confidentiality of personal information. Before sampling and performing the study, permission to conduct the study was granted by university ethics committee. 
Citation: Mohamadirizi S, Shaygannejad V, Mohamadirizi S, Mohamadirizi M (2016) Eating Disorders in a Multiple Sclerosis Clinical Population and its Association with Social Anxiety. J Mult Scler 3: 183. doi:10.4172/2376-0389.1000183

Page 3 of 4

\section{Data Analysis}

Data was analysed by using SPSS statistical software version 16 . For statistical analysis, descriptive statistics (absolute and relative frequency, mean and standard deviation) and inferential statistics (independent t-test, analysis of variance and Pearson) to investigate the relationship between gender, marital status, income, level of education, job, duration of disease with social anxiety and eating disorder score were used. $\mathrm{P} \leq 0.05$ was considered as statistical significant level.

\section{Results}

In the present study, most subjects were female (89\%), single (26\%), with non-academic education (44\%) and enough income (63\%). Also, $55.4 \%$ of subjects received interferon $\beta$ as their main treatment. Other demographic and disease characteristics are presented in Table 1.

In addition, in the study, mean and standard deviation of the social anxiety disorder score and eating disorder score were $17.9 \pm 8.5$ and $1.2 \pm 0.15$, respectively (Table 2 ). The results of Pearson correlation coefficient indicated a significant relationship between the eating disorder score and the social anxiety disorder score in total, and among the scores on each of their dimensions $(r=0.4, \mathrm{P} \leq 0.05)$ (Table $3)$.

\begin{tabular}{|c|c|c|}
\hline Variables & Mean & SD \\
\hline Age & 33.96 & 9.5 \\
\hline Night sleep & 6.9 & 1.8 \\
\hline Day sleep & 1.3 & 1.2 \\
\hline Exercise in a week & 4.3 & 1.3 \\
\hline MS disease duration & 24.3 & 6.3 \\
\hline
\end{tabular}

Table 1: Mean and standard deviation of age, night sleep time, day sleep time, exercise during a week and MS disease duration in subjects.

\begin{tabular}{|c|c|c|c|c|}
\hline \multirow{2}{*}{ Variables } & \multicolumn{2}{|c|}{ With no disorder } & \multicolumn{2}{c|}{ With disorder } \\
\cline { 2 - 5 } & $\mathrm{n}$ & $\%$ & $\mathrm{n}$ & $\%$ \\
\hline Social anxiety & 128 & 60.9 & 82 & 39.1 \\
\hline Eating disorder symptoms & 195 & 92.8 & 15 & 7.2 \\
\hline
\end{tabular}

Table 2: Frequency distribution of subjects by social anxiety disorder symptoms and eating disorder symptoms.

\begin{tabular}{|c|c|c|c|c|c|c|c|}
\hline Variable & Eating disorder dimensions & & $\begin{array}{l}\text { Body shape } \\
\text { concern }\end{array}$ & $\begin{array}{l}\text { Body weight } \\
\text { concern }\end{array}$ & $\begin{array}{l}\text { Eating } \\
\text { concern }\end{array}$ & $\begin{array}{c}\text { Dietary } \\
\text { restraint }\end{array}$ & $\begin{array}{l}\text { Total } \\
\text { score }\end{array}$ \\
\hline \multirow{8}{*}{ Anxiety dimension } & \multirow{2}{*}{ Fear } & rvalue & 0.43 & 0.34 & 0.38 & 0.36 & 0.39 \\
\hline & & $p$ value & 0.03 & 0.02 & 0.02 & 0.01 & 0.004 \\
\hline & \multirow{2}{*}{ Avoidance } & $r$ value & 0.37 & 0.39 & 0.28 & 0.34 & 0.4 \\
\hline & & $p$ value & 0.05 & 0.04 & 0.03 & 0.02 & 0.004 \\
\hline & \multirow{2}{*}{ Physiological problem } & $r$ value & 0.13 & 0.42 & -0.39 & 0.4 & 0.41 \\
\hline & & $p$ value & 0.02 & 0.02 & 0.03 & 0.02 & 0.004 \\
\hline & \multirow{2}{*}{ Total score } & $r$ value & 0.32 & 0.41 & 0.39 & 0.41 & 0.4 \\
\hline & & $p$ value & 0.04 & 0.01 & 0.03 & 0.05 & 0.05 \\
\hline
\end{tabular}

Table 3: Relationship between the eating disorder score and the social anxiety disorder score in total and among the scores on each of their dimensions.

Also, in the present study, the relationship between clinical and demographic variables and the social anxiety score was examined. The results showed that there was a significant relationship between social anxiety and Education level $(\mathrm{p}=0.03)$ and between social anxiety and exercise hours in a week $(\mathrm{r}=0.02, \mathrm{p}=0.005)$.

\section{Discussion and Conclusions}

In the present study, a majority of patients with multiple sclerosis (39.1\%) suffered from social anxiety disorder, consistent with the results from a study by Fisk et al. [21]. It should be noted that multiple sclerosis is a debilitating, progressive and unpredictable disease and causes considerable anxiety in patients after threatening health, which may be explained by biobehavioral approaches, in which anxiety is considered as endogenous production against risk stimuli and etiology theory, in which anxiety is considered a natural reaction to threatened life [22].

In addition, $7.2 \%$ of subjects suffered from eating disorder. Due to the fact that subjects in the study had other underlying chronic disease, treatment and care were likely to be affected by eating disorder. Therefore, health care team is expected to pay more attention to other life aspects of these individuals including psychological aspects. The results from the study are confirmed by a study conducted by Hossein et al. [23] where $49.7 \%$ of patients with cancer had eating disorder. In a study by Jones [24], who used the Diagnostic Survey for Eating Disorders (DSED), Eating Disorder Inventory (EDI) and Eating Attitudes Test (EAT-26), and in another study by Markowitz [25], who used the 32-item EDE-Q, the risks of the development of eating disorders in people with type 1 diabetes, were $10 \%$ and $27.5 \%$, respectively. 
As for the main objective of the study, i.e., the relationship between the eating disorder score and the social anxiety score, the results of Pearson correlation coefficient indicated that there was a significant direct relationship between these two variables. The results are supported by the results from a study by Chery et al., who reported a significant relationship between social anxiety and eating disorder. Also, the results are consistent with a study performed by Angell et al., who reported the direct and medium relationship of body image dissatisfaction, weight concern and shape concern with social anxiety [12].

It is worth to mention that like other studies, the present study has strengths and weaknesses. The study is one of few studies that examined eating disorder and social anxiety in multiple sclerosis patients, using the Social Anxiety Inventory and Eating Disorder Examination Questionnaire (EDE-Q). One of limitations of the study was its cross-sectional design, which made the determination of causality impossible. In addition, in the study, changes in weight were not examined before and during disease duration due to implementation problems. One of its other limitations was not to evaluate family's and friends' attitude towards nutritional intake and the disease as well as their history of having one of types of eating disorders because of the high number of questions, time limits and patient fatigue. So, next researchers are recommended to consider the above in their studies as much as possible.

In general, the results obtained provide evidence of eating disorder symptom conditions and their association with social anxiety in multiple sclerosis patients. Considering the findings from the study, health care team can have a broad understanding of the conditions of these two eating and social anxiety disorders, as well as the type and intensity of the relationship of the two variables, and consider both disorders in their evaluations. In addition, early diagnosing of and intervention in eating disorder and social anxiety disorder symptoms can prevent from progressing undesirable, usually severe disorder symptoms, and emerging the situations having negative effect on the patient. Health care team as individuals who spend most of their time with patients has an ideal situation to manage challenge facing patients with MS; enhancing their understanding of the relationship, they can promote these patients' life quality through designing an efficient treatment and care program.

\section{References}

1. Haghanid A, Mohammadkhan KS, Memarian R, Baharlou R (2012) The effect of peer group education program on anxiety of patients with multiple sclerosis. Iranian Journal of Medical Education 4: 249-257.

2. Mohammdi RN, Afshar H (2009) A determination of psychological and social factors creating stress in patients with multiple sclerosis. Scientific Journal of Fundamentals of Mental Health 4: 305-310.

3. Golden NH (2003) Eating disorders in adolescence and their squeal. Best Pract Res Clin Obstet Gynaecol 17: 57-73.

4. Goebel Fabbri AE (2009) Disturbed eating behaviors and eating disorders in Type 1 diabetes: Clinical significance and treatment recommendations. Psychosocial Aspects 9: 133-139.

5. Grave RD (2011) Eating disorders: Progress and challenges. Eur J Intern Med 22: 1560-1563.
6. Pourghassem GB, Hamed BMS, Kooshavar D (2010) Relationship of body mass index with eating attitude in Tabrizian high school girls. Med J Tabriz Univ Med Sci.

7. Fitzsimmons EE, Bardone-Cone AM (2011) Coping and social support as potential moderators of the relation between anxiety and eating disorder symptomatology. Eating behaviors 12: 21-28.

8. Kaye WH, Bulik CM, Thornton L, Barbarich N, Masters K (2004) Comorbidity of anxiety disorders with anorexia and bulimia nervosa. Am J Psychiatry 161: 2215-2221.

9. Hinrichsen H, Wright F, Waller G, Meyer C (2003) Social anxiety and coping strategies in the eating disorders. Eat Behav 4: 117-126.

10. Merikangas KR, Avenevoli S, Acharyya S, Zhang H, Angst J (2002) The spectrum of social phobia in the Zurich cohort study of young adults. Biol Psychiatry 51: 81-91.

11. Wonderlich-Tierney AL, Vander Wal JS (2010) The effects of social support and coping on the relationship between social anxiety and eating disorders. Eat Behav 11: 85-91.

12. Thompson AM, Chad KE (2002) The relationship of social physique anxiety to risk for developing an eating disorder in young females. J Adolesc Health 31: 183-189.

13. Celikel FC, Cumurcu BE, Koc M, Etikan I, Yucel B (2008) Psychologic correlates of eating attitudes in Turkish female college students. Compr Psychiatry 49: 188-194.

14. Bas M, Kiziltan G (2011) Relations among weight control behaviors and eating attitudes, social physique anxiety and fruit and vegetable consumption in Turkish adolescents. Adolescence-San Diego 42: 167.

15. Carter AS, Baker CW, Brownell KD (2000) Body mass index, eating attitudes and symptoms of depression and anxiety in pregnancy and the postpartum period. Psychosom Med 62: 264-270.

16. Momeni A (2005) A study of the effectiveness of the desensitization therapy method by eye movement and its reprocessing for treating social phobia. MA Clinical Psychology Thesis, Shahed University.

17. Melyani M, Shairi MR, Ghaedi G, Bakhtirari M, Tavali (2009) The effectiveness of cognitive-behavioral group therapy based on heimbergs model on the decrease of social anxiety symptoms. Iranian journal of psychaitry and clinical psychology 15: 42-49.

18. Afzal MH, Fathi Ashtiani A, Azadfallah P (2009) Evaluation of defence styles and mechanisms in patients with obsessive-compulsive disorder, generalized anxiety disorder and social phobia disorder. J Clin Psychol 1: 79-93.

19. Reas DL, Rosenvinge J (2012) The impact of age and BMI on Eating Disorder Examination Questionnaire (EDE-Q) scores in a community sample. Eating Behaviors 13: 158-161.

20. Mond JM, Hay PJ, Rodgers B, Owen C, Beumont PJ (2004) Validity of the eating disorder examination questionnaire (EDE-Q) in screening for eating disorders in community samples. Behaviour Research and Therapy 42: 551-567.

21. Poder K, Ghatavi K, Fisk JD, Campbell TL, Kisely S, et al. (2009) Social anxiety in a multiple sclerosis clinic population. Mult Scler 15: 393-398.

22. Khanjani $Z$ (2012) Effective psychological factors in multiple sclerosis: personality traits, depression, anxiety and mental stress. Tabriz University of Medical Sciences 4: 60-67.

23. Hossein SA, Bahrami M, Mohamadirizi S, Paknaad Z (2015) Investigation of eating disorders in cancer patients and its relevance with body image. Iran J Nurs Midwifery Res 20: 327-333.

24. Markowitz T (2008) Body mass index and disordered eating in adolescent females with Type 1 Diabetes. PhD Thesis, Drexel University.

25. Jones J (2000) Eating disorders in adolescent females with Type 1 diabetes mellitus: A controlled three-site study. PhD Thesis, University of Toronto. 